C-A/AP/\#234

April 2006

\title{
AGS CNI Polarimeter Data Analysis for the 2005 Run
}

\author{
J. Wood ${ }^{2}$, I. Alekseev ${ }^{1}$, A. Bravar ${ }^{2}$, G. Bunce ${ }^{2,5}$, S. Dhawan ${ }^{3}$, R. Gill ${ }^{2}$, \\ H. Huang ${ }^{2}$, W. Lozowski ${ }^{4}$, Y. Makdisi ${ }^{2}$ I. Nakagawa ${ }^{5}$, S. Rescia ${ }^{2}$, T. Roser ${ }^{2}$, D. \\ Svirida $^{1}$, K. Yip ${ }^{2}$, and A. Zelenski ${ }^{2}$
}

(1) Institute for Theoretical and Experimental Physics, Moscow, Russia

(2) Brookhaven National Laboratory, Upton, New York USA

(3) Yale University, New Haven, CT USA

(4) Indiana University Cyclotron Facility, Bloomington, USA

(5) RIKEN BNL Research Center, Upton, USA

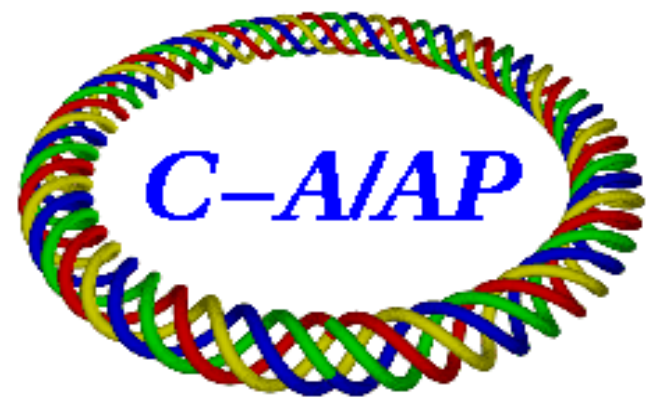

Collider-Accelerator Department

Brookhaven National Laboratory

Upton, NY 11973 


\title{
AGS CNI Polarimeter Data Analysis for the 2005 Run
}

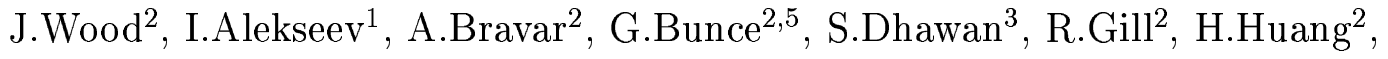 \\ W.Lozowski ${ }^{4}$, Y.Makdisi $^{2}$, I.Nakagawa ${ }^{5}$, S.Rescia ${ }^{2}$, T.Roser ${ }^{2}$, D.Svirida ${ }^{1}$, \\ K.Yip ${ }^{2}$, and A.Zelenski ${ }^{2}$ \\ (1) Institute for Theoretical and Experimental Physics, Moscow, Russia \\ (2) Brookhaven National Laboratory, Upton, USA \\ (3) Yale University, New Haven, USA \\ (4) Indiana University Cyclotron Facility, Bloomington, USA \\ (5) RIKEN BNL Research Center, Upton, USA
}

Since its installation prior to the 2003 polarized proton run, the AGS protoncarbon Coulomb-Nuclear Interference (CNI) polarimeter has been and continues to be the primary source of beam polarization measurements at AGS extraction energy. The AGS CNI polarimeter also provides the capability to measure beam polarizations at several energies in the AGS energy range from $2.4 \mathrm{GeV}$ to 24.3 $\mathrm{GeV}$, including continuous measurement of spin-dependent asymmetries during the AGS energy ramp. This report gives a brief description of the setup and general data analysis technique used for the AGS CNI polarimeter. In addition, specific details of the analysis of polarimeter data from the 2005 run are discussed. These include analysis of the energy corrections applied to the data, beam intensity and event rate effects, and study of asymmetries measured during the AGS energy ramp. 


\subsection{Setup and Detector Acceptance}

The AGS CNI polarimeter makes use of proton-carbon, $p C$, elastic scattering at very low momentum transfer squared, $-t$, in the Coulomb-Nuclear Interference region. During measurements, a very thin $\left(3-5 \mu \mathrm{g} / \mathrm{cm}^{2}\right)$ carbon target is inserted into the circulating polarized proton beam. Recoil carbon nuclei near $90^{\circ}$ from elastic scattering are detected with silicon detectors, and a left-right asymmetry, $\varepsilon$, is calculated. In order to obtain polarization information from this process, the $p C$ elastic scattering analyzing power, $A_{N}$, must be known from previous measurement. The beam polarization is given by $P \approx \varepsilon / A_{N}$. The analyzing power for $p C$ elastic scattering has been measured in experiment E950 at a beam energy of $21.7 \mathrm{GeV}$ [1]. The bulk of the CNI polarimeter measurements are made at the AGS extraction energy of $24.3 \mathrm{GeV}$. The effective analyzing power used at this energy is based on a fit to the E950 data [2]. The uncertainty in the E950 analyzing power measurement results in a large relative uncertainty in the beam polarization of approximately $\pm 30 \%$.

The targets used for the AGS CNI polarimeter are very thin carbon ribbon targets developed at the Indiana University Cyclotron Facility [3]. These targets have thicknesses of $3-5 \mu \mathrm{g} / \mathrm{cm}^{2}$. The targets are $5 \mathrm{~cm}$ long and have widths ranging from $70 \mu \mathrm{m}$ to $600 \mu \mathrm{m}$. Up to four different carbon targets can be mounted on a moveable frame inside the AGS CNI polarimeter chamber. All the targets that were used during the 2005 run measure approximately $3.6 \mu \mathrm{g} / \mathrm{cm}^{2}$ thick by $590 \mu \mathrm{m}$ wide.

The silicon detectors used to detect recoil carbon ions are segmented into 12 individual strips. Each strip measures $2 \mathrm{~mm}$ wide by $10 \mathrm{~mm}$ long. The length of the strips are oriented perpendicular to the beam direction. These silicon strip 
detectors (SSDs) are mounted in the polarimeter vacuum chamber located in section C-15 of the AGS. The proton beam interacts with the carbon target near the center of the chamber. The detectors are positioned directly to the left and to the right of the target at a distance of $32 \mathrm{~cm}$. The setup for the 2005 run used four SSDs, two in the left arm and two in the right arm.

The two detectors in each arm are positioned to have the same acceptance of carbon recoil angle, but are slightly offset in azimuthal angle. An example of the event distribution measured by the SSDs is shown in Figure 1.1. In the kinematic range of interest $\left(-t<\sim 0.05, E_{\text {recoil }}<\sim 2 \mathrm{MeV}\right)$, the distribution of recoil angles from $p C$ elastic scattering at $E_{\text {beam }}=24.3 \mathrm{GeV}$ is very narrow. The full width of the distribution is approximately $0.3^{\circ}$, which corresponds to a single SSD strip. The measured event distribution is actually much broader. This is due to the multiple scattering of recoil carbons as they exit the target. Despite these multiple scattering effects, the bulk of the event distribution falls within the SSD acceptance. At lower beam energies in the AGS, the recoil angle distribution is shifted more forward. Figure 1.2 shows a typical event distribution for $4 \mathrm{GeV}$ $<E_{\text {beam }}<5 \mathrm{GeV}$.

The SSDs have a thickness of about $400 \mu \mathrm{m}$; this is sufficient to completely stop recoil carbon ions of energies up to $\sim 100 \mathrm{MeV}$. Alpha particle sources (americium-241) mounted inside the polarimeter vacuum chamber were used for calibrating the SSDs. The $5.5 \mathrm{MeV}$ alpha particles from the sources are also completely stopped in the detectors. Incident carbons and alphas create current pulses, which are proportional to the energy deposited in the silicon. However, the silicon has an inactive, or dead, region, in which the deposited energy cannot be measured. A technique to correct for the energy loss in this dead layer has been developed and is described in Appendix A. 

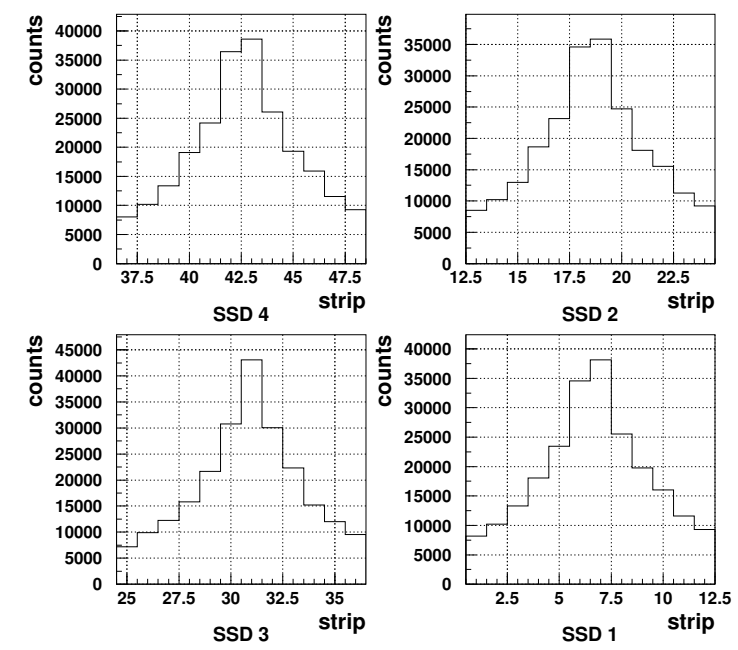

Figure 1.1: Measured event distribution vs. SSD strip number at $E_{\text {beam }}=24.3$ $\mathrm{GeV}$. Four detectors were used for each measurement during the 2005 run. SSD 1 and 2 were positioned to the right of the beam, on the outer radius of the AGS. SSD 3 and 4 were positioned to the left, on the inner radius. The strip number gives some indication of the recoil angle distribution; however, the recoil angle is smeared by multiple scattering of the carbon as it exits the target. 

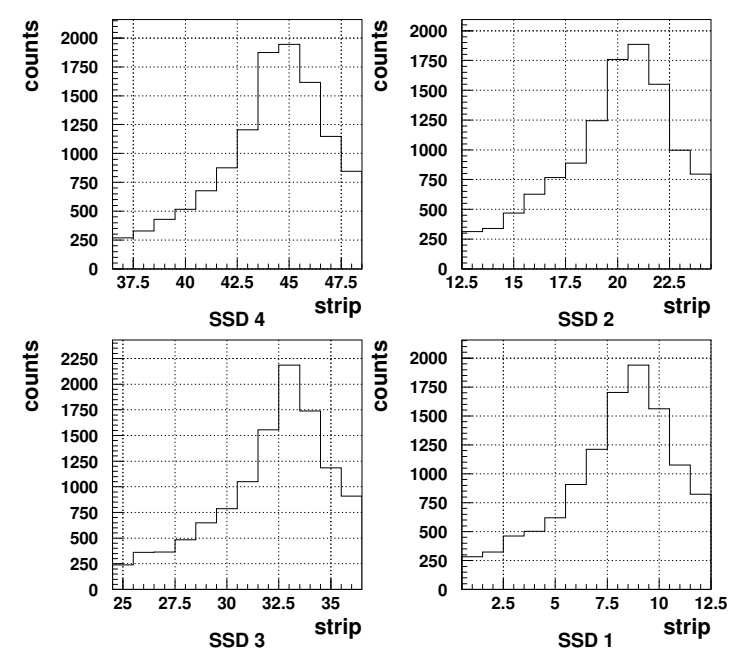

Figure 1.2: Measured event distribution vs. SSD strip number for $4 \mathrm{GeV}$ $<E_{\text {beam }}<5 \mathrm{GeV}$. At lower beam energies, the distribution shifts more forward. However, much of the distribution is still within the detector acceptance.

\subsection{Basic Analysis Method}

The current pulses from the SSDs are processed by the polarimeter data acquisition (DAQ) system. After passing through a series of amplifiers, event pulses are analyzed by a waveform digitizer (WFD) system [4]. The WFD modules extract timing, amplitude, and integrated charge from each event pulse. The AGS beam energy, RF bucket position, and polarization direction of the beam are also available for each event. A system of criteria use this information to separate carbon events from background and to ensure overall data quality.

The main criterion used to separate scattered carbon events from background is based on the carbon time of flight $(t o f)$ to kinetic energy $\left(E_{k i n}\right)$ correlation. The kinematics of the recoil carbons that are of interest $(<\sim 2 \mathrm{MeV})$ can be 
described non-relativistically.

$$
t o f=l \sqrt{\frac{m_{C}}{2 E_{k i n}}},
$$

where $l=32 \mathrm{~cm}$ is the distance from the target to the detector and $m_{C}$ is the carbon mass, $11.18 \mathrm{GeV} / c^{2}$. The event cut is set to select events between $\pm 20 \mathrm{~ns}$ of the expected tof-energy correlation. Figure 1.3 shows a graphical representation of the event selection.

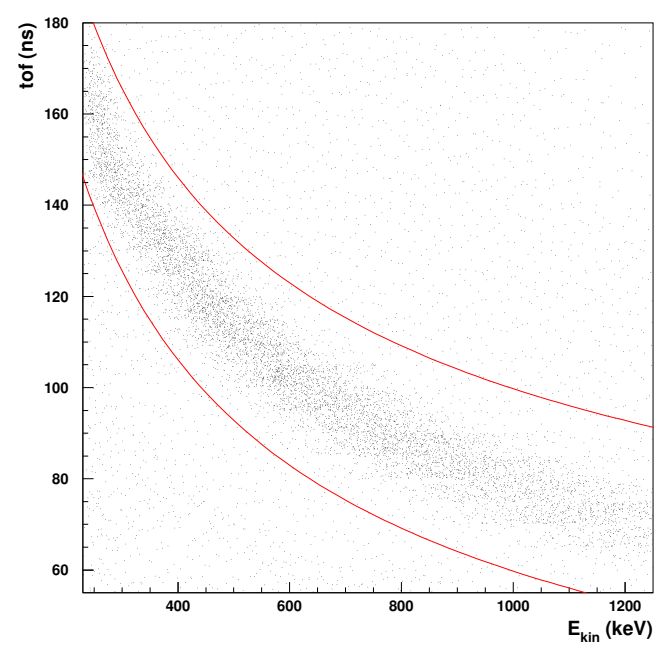

Figure 1.3: Time of flight vs. kinetic energy of detected events from a polarimeter measurement. The lines represent the expected tof-energy correlation (Equation 1.1) \pm 20 ns. Only events between the two lines are selected for calculating asymmetries.

In addition to the tof-energy correlation cut, events were also selected for a kinematic range in momentum transfer. For $p C$ elastic scattering, the momentum transfer squared, $-t$, is proportional to the kinetic energy of the scattered carbon,

$$
-t=2 m_{C} E_{k i n}
$$


where $m_{C}$ is the carbon mass and $E_{k i n}$ is the kinematic energy of the scattered carbon. During the 2003 running period, the kinematic range used for calculating asymmetries was defined to be $0.009(\mathrm{GeV} / c)^{2} \leq-t \leq 0.022(\mathrm{GeV} / c)^{2}$, which corresponds to approximately $400 \mathrm{keV} \leq E_{k i n} \leq 1000 \mathrm{keV}$. This same range was used for polarization measurements during the 2004 and 2005 runs. The events that pass the selection cuts are used to calculate the asymmetry, $\varepsilon$. An effective analyzing power, $\left\langle A_{N}\right\rangle$, is also calculated for the specific $-t$ range. Beam polarization is then given by $P=\varepsilon /\left\langle A_{N}\right\rangle$. $\varepsilon$ and $\left\langle A_{N}\right\rangle$ are defined in Appendix B.

\subsection{Data Corrections}

A system of "on-line" analysis software is used to quickly provide asymmetry and polarization values for each polarimeter measurement. This on-line analysis method applies corrections to measured amplitude and time to reconstruct the incident kinetic energy and tof for each event. The energy correction adjusts the measured energy to account for energy loss in the silicon dead layer. The scale of this correction is parametrized in terms of the dead layer thickness, $t_{\text {dead }}$. The energy correction method has recently been studied and refined. Analysis with this new correction method is referred to as the "off-line" analysis. The results of the off-line analysis and a discussion of the changes between the on-line and off-line methods are described in this section.

\subsubsection{Other parameterizations for energy correction}

All corrections made to the measured carbon energy are assumed to be due to energy lost in the silicon dead layer. However, there is evidence that energy 
corrections may be compensating for other effects that are not related to the dead layer. The scale of the energy correction appears to depend on the preamplifier electronics that are connected to the SSDs. This effect was first noticed during the 2003 run. At that time, the polarimeter used only two SSDs. Each 12channel detector was connected to two 6-channel pre-amp circuit boards. Figure 1.4 shows an example of the energy corrections extracted from fitting the carbon tof- $E_{k i n}$ correlation for each strip. For a single detector the energy correction is $\sim 20 \mathrm{keV}$ different for different pre-amp boards. If the corrections are solely due to the dead layer, then the corrections are expected to be much more uniform across the detector. This is because the dead layer thickness is expected to be the same for all strips in a single detector.
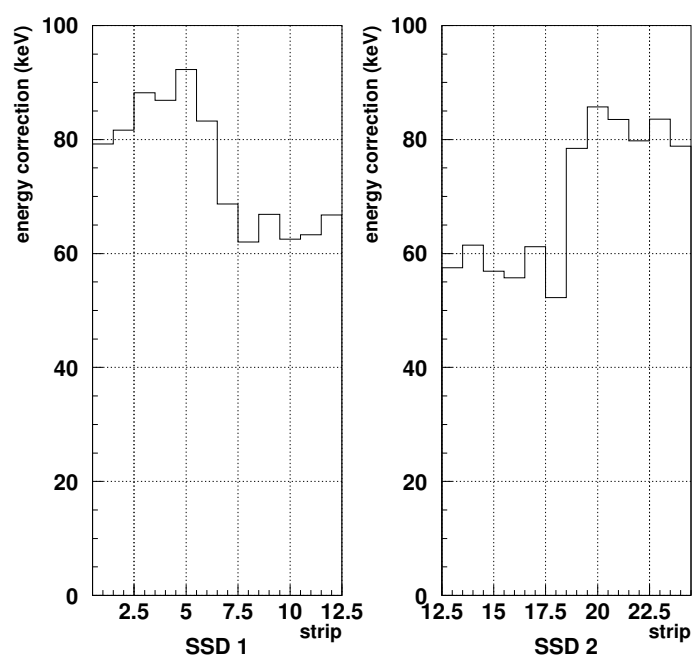

Figure 1.4: Energy correction versus SSD strip number for the two SSDs used during the 2003 run.

During the 2004 run, further evidence for the energy correction dependence on the pre-amp boards was discovered. Four SSDs were used in the polarimeter for this run, and each SSD was connected to a 12-channel pre-amp board. One 
Table 1.1: Average energy correction parameter for each detector used during the 2004 run. The energy correction is parameterized in terms of the dead layer thickness, $t_{\text {dead }}\left(\mu \mathrm{g} / \mathrm{cm}^{2}\right)$.

\begin{tabular}{|l|c|c|c|c|}
\hline & SSD 1 & SSD 2 & SSD 3 & SSD 4 \\
\hline Set A & 43.2 & 49.3 & 27.2 & 46.4 \\
\hline Set B & 47.5 & 53.0 & 23.2 & 45.7 \\
\hline
\end{tabular}

of the detectors, SSD 3, had significantly smaller energy corrections than the other three. Midway through the run, the four SSDs were removed, and a new set of four detectors were installed. Again, energy corrections for SSD 3 were anomalously low. (See Table 1.1.) This suggests that some effect, not associated with the detector, is influencing the energy corrections.

The energy corrections for the 2005 data have recently been studied. The energy correction is described in terms of the extracted value of $t_{\text {dead }}$. Details of the method used to extract $t_{\text {dead }}$ from the data can be found in Appendix A. Approximately $5 \%$ of the $\sim 1400$ individual measurements were used to extract $t_{\text {dead }}$. This gave a good indication of the behavior of the energy correction throughout the history of the run. For all the detectors used during the 2005 run, the values of $t_{\text {dead }}$ increased as the run progressed. Figure 1.5 shows the average value of $t_{\text {dead }}$ for one SSD versus the total number of events accumulated by the polarimeter. All detectors showed similar increases of $10-15 \mu \mathrm{g} / \mathrm{cm}^{2}$ during the run. This large increase is further evidence that the dead layer correction is compensating for some other effect(s). Such a large increase in the actual dead layer thickness does not seem plausible.

Evidence for non-dead layer type effects in the energy correction of polarimeter 


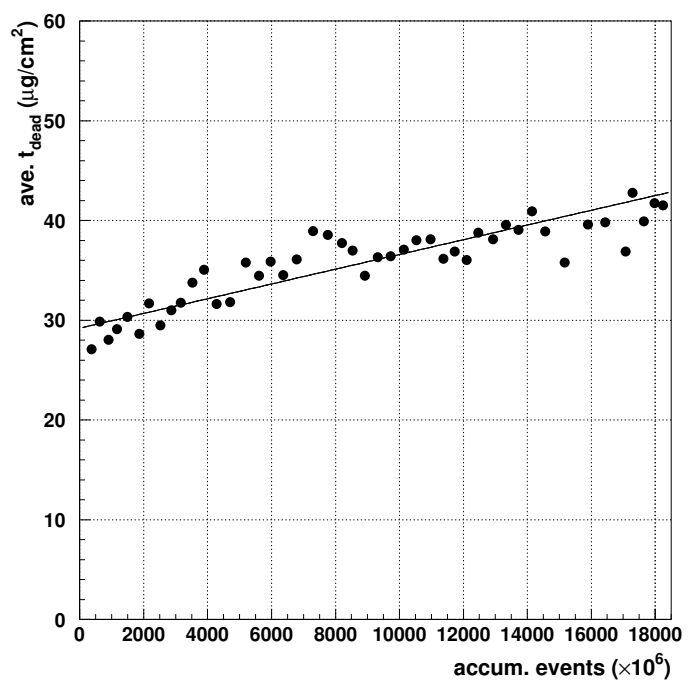

Figure 1.5: Average value of $t_{\text {dead }}$ for one SSD versus total events accumulated by the polarimeter DAQ system.

data led to studies of alternate parametrizations for the corrections. As described in Appendix A, the current data corrections are parametrized in terms of one energy correction parameter, $t_{d e a d}$, and one time correction parameter, $t_{0}$. Several new parameterizations of the energy correction were tried; however, fits to the data frequently did not converge to reasonable values when using more than one energy correction parameter. This limited the energy correction to very simple forms. One form that was used to relate measured energy deposited, $E_{d e p}$, to incident kinetic energy, $E_{k i n}$, is the following,

$$
E_{k i n}=E_{c o r}+E_{d e p}
$$

where $E_{c o r}$ is the energy correction parameter. The scale of the extracted $E_{\text {cor }}$ values are around $70 \mathrm{keV}$, but they, like $t_{\text {dead }}$, also increase throughout the history of the run. Next, a fixed dead layer correction, corresponding to $t_{\text {dead }}=35 \mu \mathrm{g} / \mathrm{cm}^{2}$, was added. With a fixed dead layer correction, the values of $E_{\text {cor }}$ are only about 1 
$\mathrm{keV}$. This suggests that additional corrections beyond a dead layer correction are small. Although evidence for non-dead layer type effects exists, the dead layer correction method appears to provide sufficient correcttions to the data.

\subsubsection{Offline analysis results}

The off-line analysis method used for the 2005 data employs the dead layer correction described in Appendix A. For several individual polarimeter measurements the tof- $E_{k i n}$ correlation was fit for each strip of each detector. For each strip $t_{\text {dead }}$ and $t_{0}$ values were extracted. These extracted $t_{\text {dead }}$ and $t_{0}$ values were then used to determine the corrections for all of the approximately 1400 polarimeter measurements. The $t_{d e a d}$ values from each strip were used to find an average $t_{\text {dead }}$

for each of the four SSDs used in a particular measurement. The average was weighted by the number of events that each strip detected. Average $t_{\text {dead }}$ was plotted versus the total accumulated events during the lifetime of the detector and fit with a linear function. (See Figure 1.5). A similar fit was performed for all of the detectors used during the 2005 run. The result of these fits were used to determine the values of $t_{\text {dead }}$ to use for correcting each individual measurement. The $t_{0}$ values show less significant trends over the course of the run than the $t_{\text {dead }}$ values. The $t_{0}$ values were fit with a constant for each strip, and the constant value is used for all off-line corrections.

The main difference between the on-line and off-line correction techniques is the frequency with which the $t_{\text {dead }}$ values were updated. As described above, the off-line method determines a set of $t_{\text {dead }}$ values for every polarimeter measurement. For the on-line method, $t_{\text {dead }}$ (and $t_{0}$ ) values were only updated periodically, and the most current values were used to correct all subsequent data. Also, at times there were sizeable gaps between updates. Other differences between the on- 
line and off-line methods have only small effects on the final polarization results. These include differences in the averaging of $t_{\text {dead }}$ for a detector (numerical average vs. weighted average) and differences in the fitting techniques to extract $t_{\text {dead }}$ and $t_{0}$ from the data. The off-line method uses exactly the technique in Appendix A. The on-line method was slightly different. Some of the functional forms used to describe the energy loss behavior included higher order polynomial terms. During off-line analysis these higher order terms were deemed unnecessary.

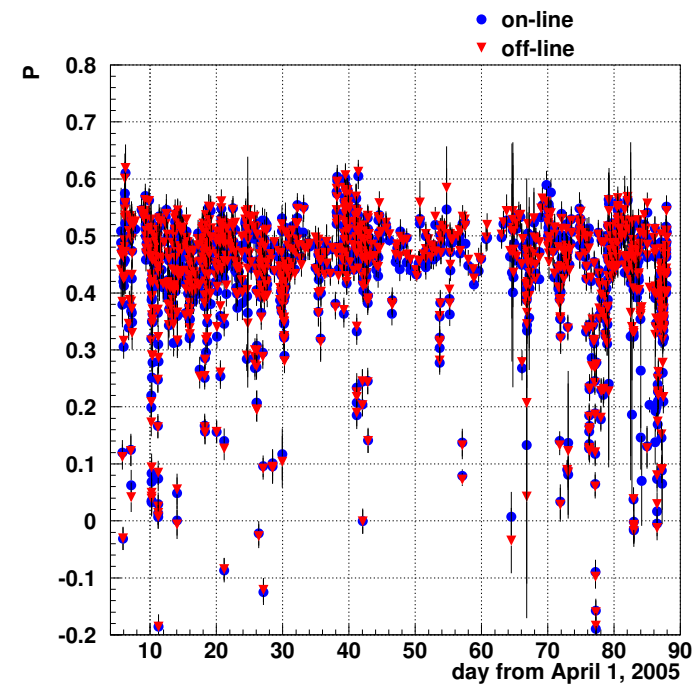

Figure 1.6: AGS polarization results versus day from April 1, 2005. The blue circles are the on-line results. The red triangles are the off-line results. The error bars represent the statistical errors of the measurements.

Much effort was put forth during off-line analysis to determine the best way to correct the measured recoil carbon energy. Nevertheless, the final results of the off-line analysis are very similar to the on-line results. The on-line and offline polarization values from the 2005 run are shown in Figure 1.6. The relative difference between the off-line and on-line polarizations $(\Delta P / P)$ is plotted in Figure 1.7. On average, the off-line polarization is slightly larger than on-line. 
Despite efforts to improve the correction technique, the final polarization does not show a very significant change. This suggests that the polarimeter does not have a strong sensitivity to the energy correction technique.

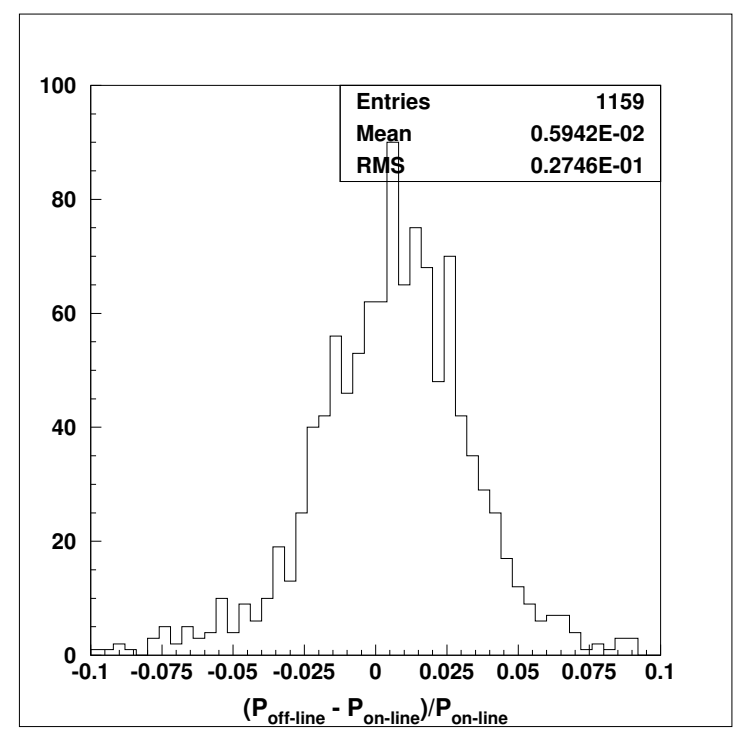

Figure 1.7: Distribution of $\Delta P / P$ between on-line and off-line values. The mean of the distribution is slightly positive, indicating an overall increase for the off-line results.

\subsection{Intensity and Event Rate Effects}

Several studies have been performed off-line in order to determine the level of systematic uncertainty in the AGS CNI polarimeter measurements. In order for the AGS CNI polarimeter to be effective, the measured asymmetries must be robust when subjected to a variety of different criteria. One issue of concern with the 2005 data is the polarimeter's dependence on beam intensity and event rate. The beam intensity and the polarimeter event rate are directly related. High beam intensity will produce a large event rate. If the event rate becomes too 
large, then event pileup can occur.

Event pileup happens when a proton bunch passes the carbon target and more than one scattered particle is incident on a single detector strip. If this occurs, the WFD algorithm only finds the event with the highest amplitude. The lower amplitude event(s) is(are) lost. If event pileup occurs frequently, then the missed events can significantly alter the measured asymmetry. The measured event rates in the AGS CNI polarimeter suggest that the probability of event pileup is small. The event occupancy (i.e. number of events per bunch passing) is estimated to be approximately $5 \%$ for the most populated detector strips. This estimate of event occupancy is based on measured event rates with beam intensity $\approx 1 \times 10^{11}$ protons per bunch. The probability of pileup increases at higher beam intensities. Measurements with a variety of different intensities have been studied to understand effects of event pileup.

Event pileup is most likely to occur in the detector strips with the highest event rates. As seen in Figure 1.1, the central strips of each detector are much more populated than strips on the edges. To determine the effect of event pileup on the measured asymmetry, asymmetries with different strip selection were studied. Asymmetries were calculated using events from only the three most populated strips from each detector. Asymmetries were also calculated with events from the outer three strips on either side of the central strips. The ratio of asymmetries for the outer and central strips are plotted versus beam intensity in Figure 1.8. As beam intensity increases event pileup will affect the asymmetry from the central strips $\left(\varepsilon_{\text {central }}\right)$ more than the asymmetry from the outer strips $\left(\varepsilon_{\text {outer }}\right)$. The data in Figure 1.8 shows that the ratio of asymmetries is not strongly dependent on beam intensity. A representative sample of measurements from the 2005 data set were selected for this study. The measurements studied show that 
there is no significant effect on the asymmetry from event pileup.

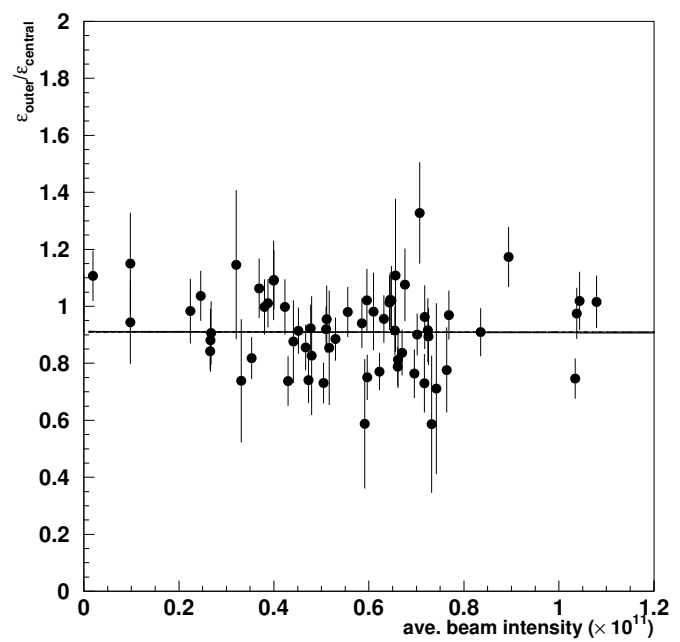

Figure 1.8: Ratio of asymmetries for the outer and central strips vs. the average beam intensity for each measurement. There is no obvious dependence on beam intensity. The line represents a constant fit to the data points. These data represent a fraction of the 2005 data set. Approximately every 20th measurement was selected for this analysis. The error bars are statistical only.

Many of the concerns about rate effects in the polarimeter have been motivated by studies of the beam polarization profile. By moving the carbon target to different horizontal positions, the polarimeter can measure polarization for different parts of the beam. One set of profile measurements that were of particular interest were made while operating the AGS with the new super-conducting partial Siberian snake magnet referred to as the "cold snake". These polarization measurements are plotted versus target position in Figure 1.9a. With the cold snake in operation, the horizontal polarization profile of the beam is expected to be a smooth curve that peaks at the center of the beam. The polarimeter measurements suggest that the profile is essentially flat for a large part of the 
beam. Another unexpected result is the event rate of the polarimeter (Figure 1.9b.) seems to plateau near the center of the beam, while the distribution of beam particles is thought to be gaussian. These results prompted further study of this data.
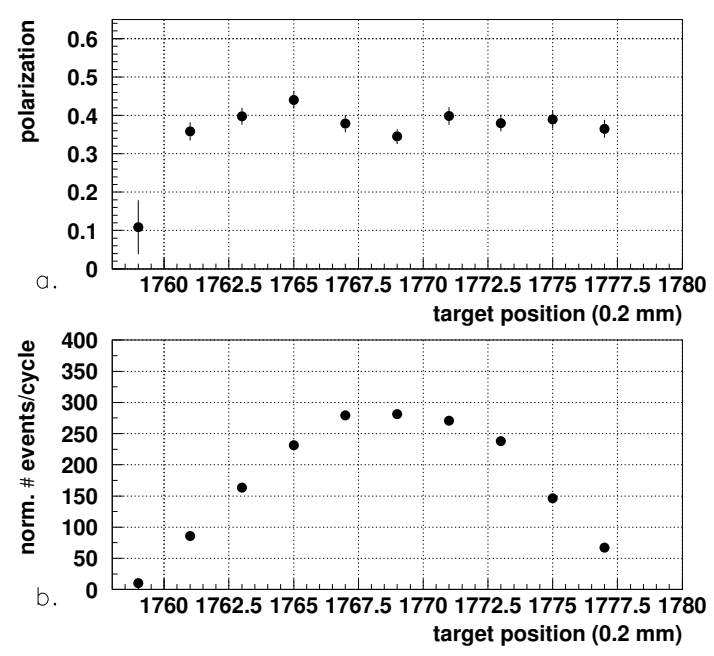

Figure 1.9: a. Measured polarization versus target position during AGS operation using only the cold snake on June 26, 2005. The error bars are statistical only. The target position are in units of $0.2 \mathrm{~mm}$. b. The normalized event rate versus target position with cold snake only. The normalized event rate is the number of polarimeter events per AGS cycle divided by the beam intensity. The numbers plotted here are averaged over the number of cycles in each measurement.

As described above, one way to determine if the polarimeter is sensitive to rate effects is to compare asymmetries from different detector strips. The ratio of polarizations for outer and central strips was calculated for the cold snake profile measurements (Figure 1.10). The ratio shows no significant dependence on the target position. Others studies were developed to look for effects from event rates in these profile measurements. These included comparing the shape of the event 
distribution vs. detector strip and comparing measured asymmetries for different carbon energy bins. None of these studies showed any significant effects from event rate.

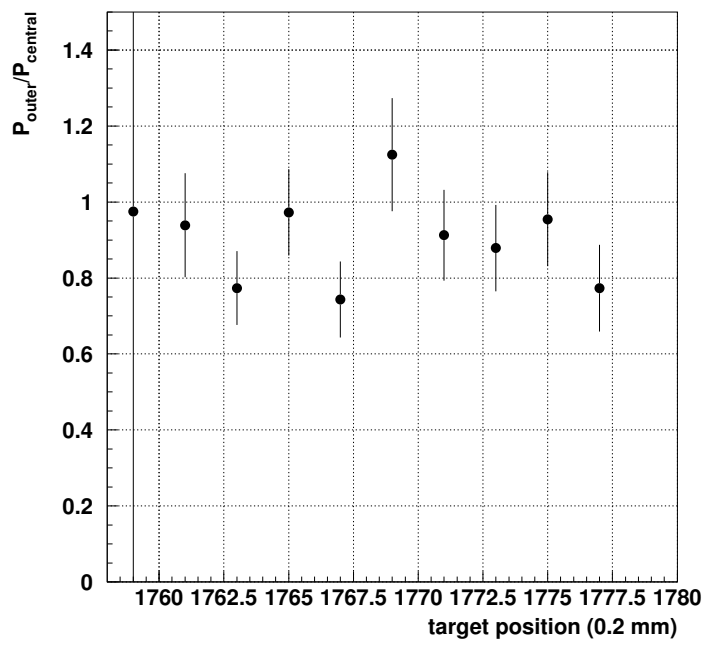

Figure 1.10: Ratio of polarizations for the outer and central strips versus target position.

The asymmetries measured by the polarimeter depend on the dead layer energy corrections that are applied to the data (described in Section 1.3 and Appendix A). If the energy corrections are influenced by the beam intensity and event rate, then this can affect the polarimeter measurements. Figure 1.11 shows the average $t_{\text {dead }}$ values for one SSD versus event rate. Some of the $t_{\text {dead }}$ data in Figure 1.11 were taken from the beam profile measurements described in the paragraphs above. The other $t_{\text {dead }}$ values in the plot were taken from measurements near the end of the 2005 running period when the beam intensity was being varied. These two data sets were chosen for this study because they cover relatively large ranges of event rates during a short time period. The data from the profile measurements do not show an obvious dependence on event rate, but 
the trend from the intensity scan data is clearer. The $t_{\text {dead }}$ values show a small increase with event rate. This suggests that beam intensity and event rate may have some influence on polarimeter measurements. However, the effect on the measured polarization from a small increase in $t_{\text {dead }}$ is minor. Previous studies have shown that a $t_{\text {dead }}$ increase of $5 \mu \mathrm{g} / \mathrm{cm}^{2}$ results in $\sim 2.5 \%$ increase (relative, not absolute) in the measured polarization.

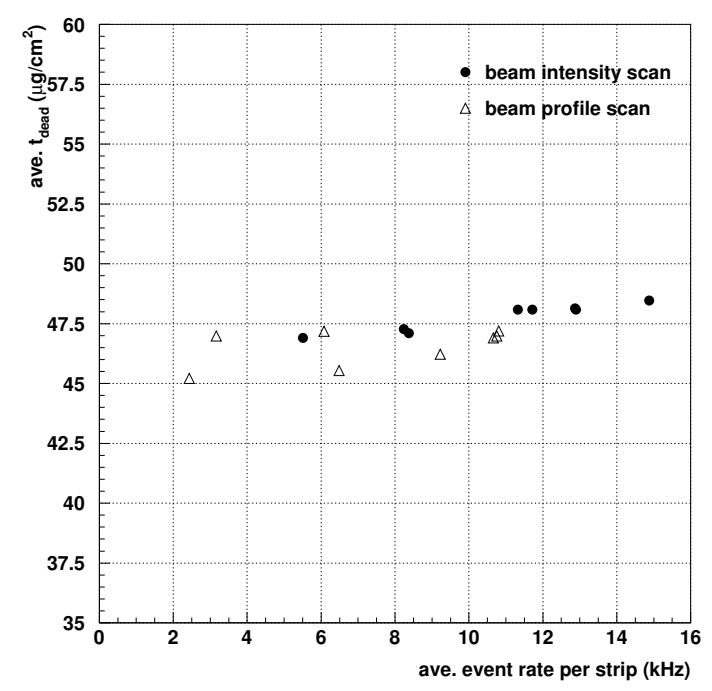

Figure 1.11: Average value of $t_{\text {dead }}$ for one SSD versus average event rate per detector strip. The event rate is averaged over all the AGS cycles for a given measurement then divided by the total number of detector strips (48). Data from the beam profile measurement (triangles) and from a beam intensity scan (circles) are shown. The fitting method used to extract $t_{\text {dead }}$ returns a very small statistical error (less than the size of the points). The actual uncertainty in $t_{\text {dead }}$ is expected to be larger, but further study is needed to quantify the error.

Another issue related to event rate and beam intensity is the large number of relativistic background events that are observed with the AGS CNI polarimeter. These events are created when the proton bunch interacts with the carbon tar- 
get, and they arrive at the detectors well before the slower moving recoil carbons. Figure 1.12 shows an example of these events. The boundary of the data acquisition time window is normally set to mask these events out of the data stream, so these background particles cannot be directly studied off-line. Further study of possible effects from these background events are planned for the future.

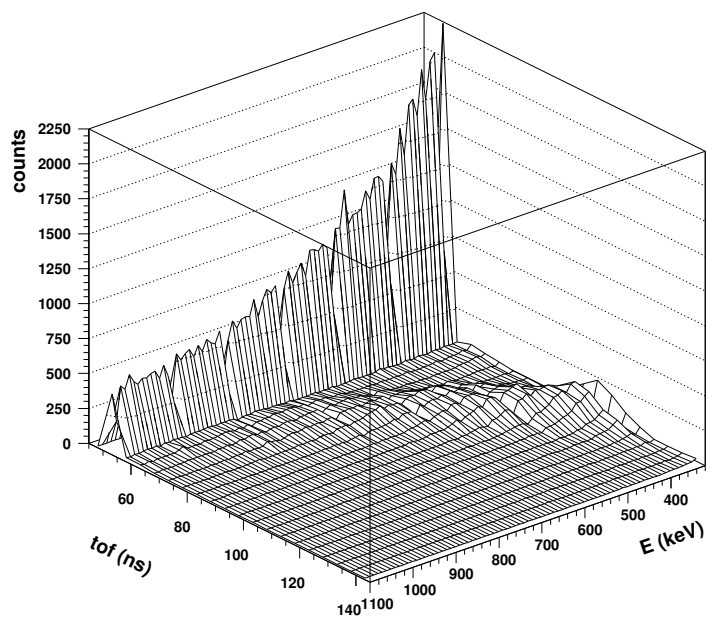

Figure 1.12: A three-dimensional surface plot of time of flight vs. energy. Many prompt background events are seen near tof $=50$ ns. The carbon events are seen later in tof. The carbon tof decreases as energy increases. For energies greater than $1000 \mathrm{keV}$, the carbon band is difficult to distinguish from the prompt background.

\subsection{Measurements during the AGS Energy Ramp}

For a typical polarization measurement, the AGS CNI polarimeter detects scattered carbon events only after the proton beam has been accelerated and held at a particular flattop energy. However, the polarimeter was also used to characterize 
the polarization of the beam as it was being accelerated. For these measurements the polarimeter target was inserted into the beam during the early part of acceleration cycle, and the target remained in the beam throughout the acceleration up to AGS extraction energy. Each detected event is associated with a "gauss clock count", which is proportional to the beam momentum. Detected events are collected into narrow momentum bins (10-50 MeV/c wide). Asymmetries are then calculated for each bin. The asymmetries provide information about the behavior of the beam polarization relative to the momentum of the beam.

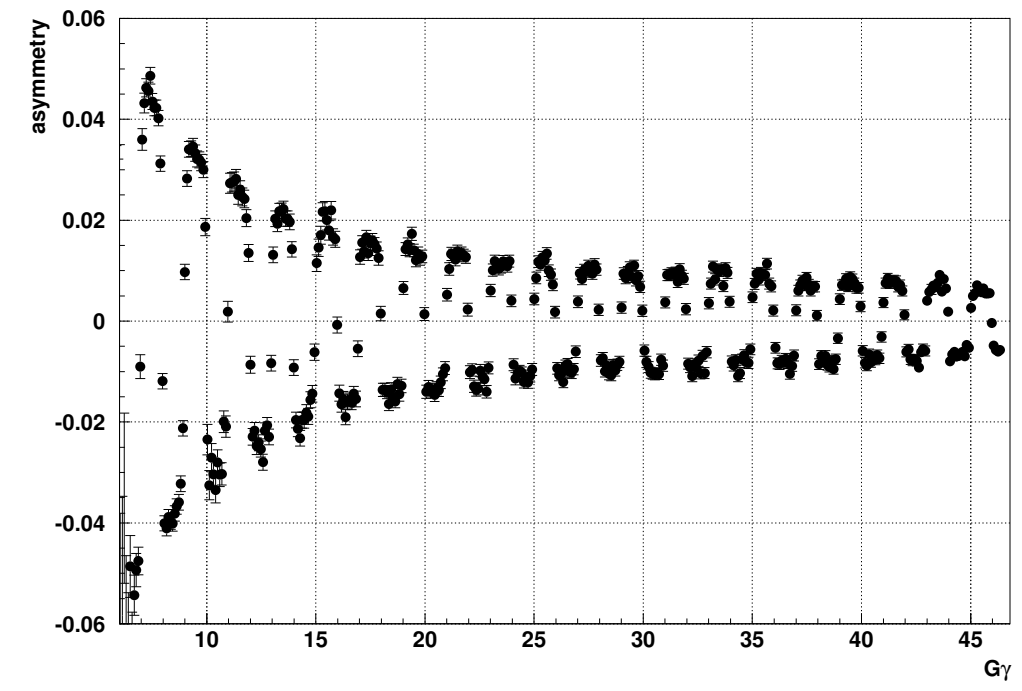

Figure 1.13: Measured asymmetry versus $G \gamma$. The sign of the asymmetry changes when resonance conditions are crossed. The error bars are statistical only. These data were accumulated during a four-hour period on June 25, 2005. The AGS was operating with the cold snake only.

Figure 1.13 shows the measured asymmetry versus the parameter $G \gamma(G \gamma \approx$ $1.9 \times$ beam energy in $\mathrm{GeV}$ ). The sign of the asymmetry changes each time $G \gamma$ is equal to an integer. The proton spin direction is expected to flip when resonance 
conditions are encountered at each $G \gamma=$ integer. These measurements clearly confirm this behavior. The data in Figure 1.13 shows that the magnitude of the asymmetry decreases as the beam energy increases. To better illustrate this dependence, the asymmetries measured during acceleration were averaged for each spin flip. Data taken during or very near a resonance crossing were not included in the average value. Data for $n-0.1<G \gamma<n+0.1$, where $n$ is an integer, were not used in the average value. The magnitude of the average $\varepsilon$ versus $G \gamma$ is shown in Figure 1.14 for three different data sets. The decrease in $\varepsilon$ is only partially due to loss of polarization as the beam is accelerated. Measurements at various beam energies in the AGS have shown that the analyzing power also decreases with beam energy. However, the exact energy dependence of the analyzing power is not known.

The data plotted in Figure 1.14 were measured under three separate running conditions. The blue points represent asymmetries measured during the 2004 running period. The AGS operated using a warm partial Siberian snake with an RF dipole magnet to correct for certain intrinsic resonance conditions. The cold snake was installed for the 2005 run and was expected to reduce effects of depolarizing resonances. The red points in Figure 1.14 show the data using both the cold snake and the warm snake, and the black points are with the cold snake only. Some of the motivations for doing these measurements are to identify specifically when in the acceleration cycle that polarization is lost and to determine if different Siberian snake configurations can improve the polarization output. The data suggests that there are no obvious differences in the polarization losses for the three different snake configurations. The three operating methods did produce slightly different polarizations after accelerating to extraction energy. The data in Figure 1.14 correspond to final polarization values of $44.9 \%$ for 


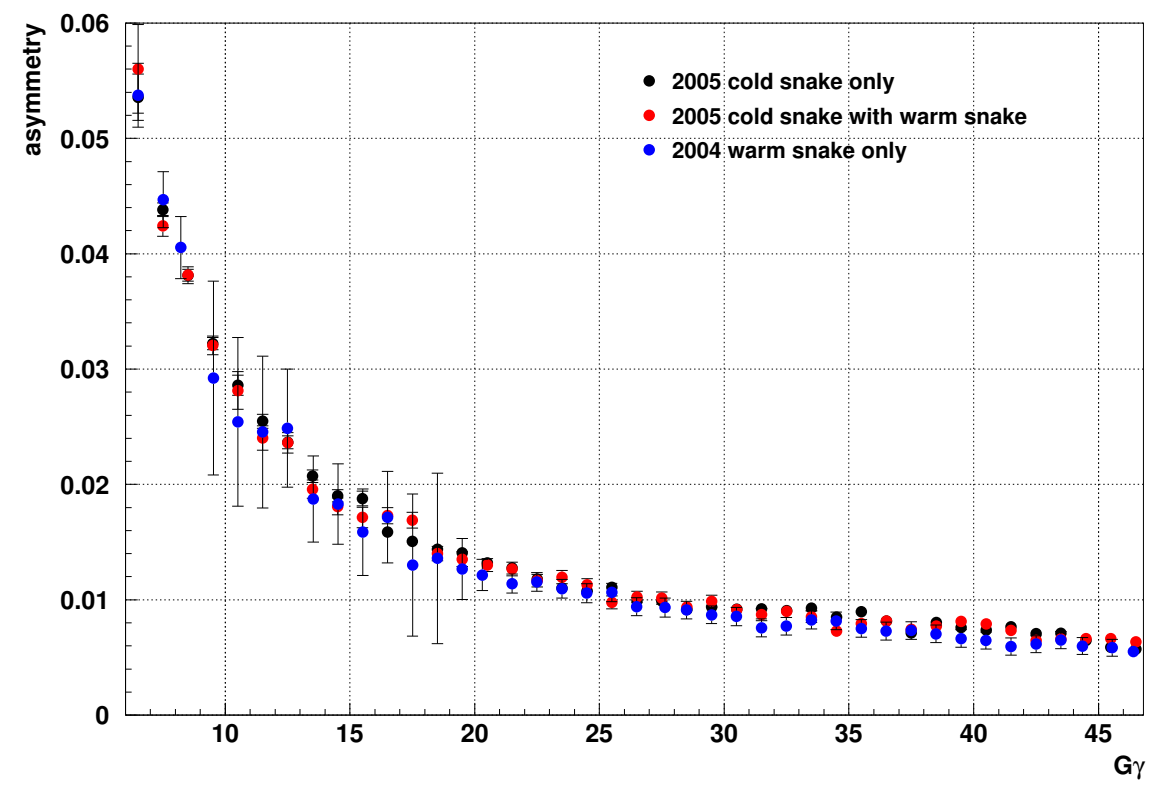

Figure 1.14: The magnitude of $\varepsilon$ versus $G \gamma$. The black points were measured during the 2005 run using only the cold snake. The red points are also from 2005. Both the cold and warm snakes were used during these measurements. The blue points are from the 2004 run using the warm snake with the RF dipole. Limited statistics were available in 2004 for $G \gamma<20$ because there was difficulty maintaining a steady beam position in the early part of the acceleration cycle. The error bars on the points are statistical. 
the 2005 cold snake only, $49.9 \%$ for the 2005 cold snake with warm snake, and $43.3 \%$ for the 2004 warm snake mode. The statistical error on the polarization is $\pm \sim 0.5 \%$.

A new analysis method has led to an improved interpretation of the asymmetries measured during acceleration using only the cold snake. Simulations of the AGS acceleration cycle with the cold snake predict that the vertical spin component of the proton, $S_{y}$, behaves as

$$
S_{y}=\frac{\sin (G \gamma \pi) \cos \left(\frac{\chi}{2} \pi\right)}{\sqrt{1-\left(\cos (G \gamma \pi) \cos \left(\frac{\chi}{2} \pi\right)\right)^{2}}}
$$

where $\chi=\left(24.4678-\frac{15.4542}{1.56058-G \gamma / 1.7928}\right) / 180^{\circ}$. A function of the form in Equation 1.4 was used to fit the measured asymmetry data. An example of a fit between two integer values of $G \gamma$ is shown in Figure 1.15. The amplitude of the fit function is then used to determine the appropriate value for the asymmetry between each $G \gamma$ integer. This technique provides a more accurate description of the asymmetry behavior than simply averaging the points between $n-0.1<G \gamma<n+0.1$. The asymmetry values from this method are slightly larger (by $\sim 4 \%$ ) than the averaging method. Currently, the model of the vertical spin component (Eq. 1.4) exists just for AGS acceleration with the cold snake only. Models for other snake configurations have not been developed

\subsection{Conclusions}

During the 2005 run, the AGS CNI polarimeter successfully made many polarization measurements. The polarimeter provided a monitor of the polarization values at AGS extraction energy and also gave useful feedback for optimizing the accelerator conditions for maximum polarization output. Before polarization values can be calculated a correction to the measured recoil carbon energy must 


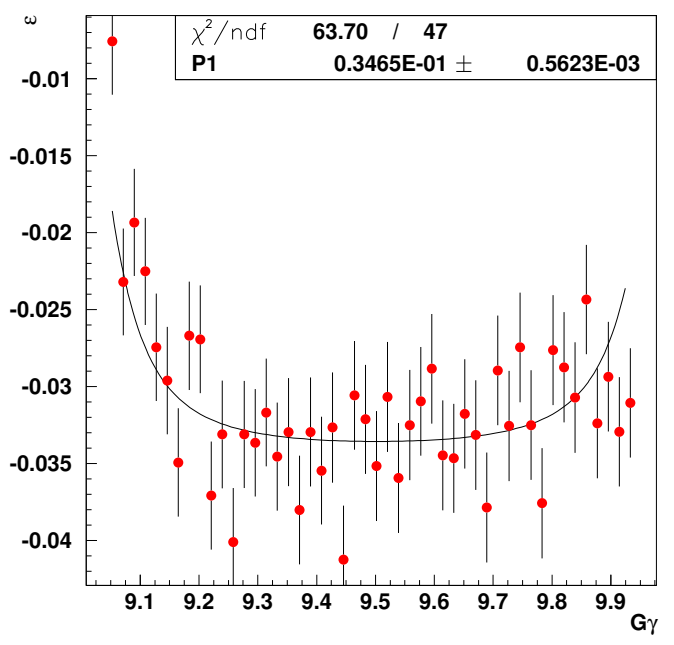

Figure 1.15: Measured asymmetries fit with the function in Equation 1.4. The error bars are statistical only. These data are fit for values of $G \gamma$ between 9.05 and 9.95. Similar fits were performed between each $G \gamma$ integer.

be applied. This correction accounts for energy lost in the dead layer of the silicon detectors. The energy correction technique has been studied extensively. Evidence suggests that the correction compensates for other effects not related to the silicon dead layer. Nevertheless, parametrizing the energy correction in terms of the dead layer thickness has been shown to be an effective technique for the AGS CNI polarimeter. During off-line analysis, the energy correction for each polarimeter measurement was updated. This provided a new set of offline polarization values. In comparison to the orignal, on-line values, the off-line polarization generally shows a small $(\sim 0.5 \%)$ increase.

Another issue that was studied off-line for the 2005 data set was the effect of beam intensity and event rate on the polarimeter. Studying measured asymmetries from different detector strips suggested that the event rate has no significant effect on the polarimeter measurements. Particular attention was paid to mea- 
surements of the horizontal polarization profile using the cold snake only in the AGS. The measured profile caused suspicion because the shape differed from expectations. Still, no significant signs of rate effects were found in this data. From previous years' data, the existence of an intensity-dependent blast of relativistic particles incident on the polarimeter detectors was discovered. These prompt particles could possibly have an effect on the polarimeter measurements. However, these events are masked out of the polarimeter data stream. So, a direct study of them is not possible with the existing data. Future studies of these events are planned.

The AGS CNI polarimeter was also used to measure asymmetries while the proton beam was being accelerated. During the 2005 run, measurements were made during the energy ramp with different configurations of the cold and warm snakes. The asymmetry measurements were compared to see if there were any differences in polarization loss for the different snake configurations. Measurements with the cold snake only and with the cold and warm snakes together were compared with measurements from the 2004 run with the warm snake and RF dipole. No obvious differences were seen between the three different sets of measurements. However, the highest polarization values at extraction energy were achieved using the cold snake together with the warm snake.

In summary, the measurements of the AGS CNI polarimeter provide valuable information for the RHIC Spin Program. The polarimeter is used as a monitor of the beam polarization injected to RHIC. The polarimeter is also used to study different accelerator conditions in order to improve the polarization output from the AGS. The energy corrections to the polarimeter data have been studied. The final polarization results do not show a strong dependence on the energy correction method. The possibility of systematic effects due to high event rates 
has been studied, and no significant effects are seen. Polarimeter data measured during the AGS energy ramp have been studied. These measurements reveal no obvious differences between the acceleration with different snake configurations. With effort the AGS CNI polarimeter will continue to provide useful diagnostic information for the advancement of the RHIC Spin program. 


\section{Appendix A: Silicon Dead Layer Energy Loss Correction}

Before event selection cuts can be applied, the measured energy of each event must be corrected to account for energy lost in the silicon dead layer. The energy correction method makes use of an energy loss $\left(\frac{d E}{d x}\right)$ table for carbon ions in silicon. A program called MSTAR [5] generates this table for carbons with incident energies from 0.1 to $2 \mathrm{MeV}$. The data from the table are fit well with a fourth order polynomial. See Figure A.1. This function is used to calculate the integrated energy loss for a dead layer of a given thickness. A thickness of 150 $\mathrm{nm}$, or $35 \mu \mathrm{g} / \mathrm{cm}^{2}$, is expected for the SSDs used for this polarimeter, but the exact value is unknown.

Energy loss is calculated for a range of dead layer thicknesses from 15 to 65 $\mu \mathrm{g} / \mathrm{cm}^{2}$. For each thickness the energy deposited in the dead layer, $E_{\text {dead }}$, is calculated for carbon incident kinetic energies between 0.1 and $2 \mathrm{MeV}$. Figure A.2 shows the calculated values of energy deposited in the active silicon, $E_{d e p}$ $\left(=E_{k i n}-E_{\text {dead }}\right)$, plotted versus $E_{k i n}$ for several values of $t_{\text {dead }}$. For each thickness the points are fit with a second order polynomial from $350 \mathrm{keV}$ to $1100 \mathrm{keV}$ in $E_{\text {dep }}$.

Again, the goal of this method is to be able to correct the experimentally measured values of $E_{d e p}$ to produce the initial kinetic energy, $E_{k i n}$. The fits in Figure A.2 relate $E_{d e p}$ to $E_{k i n}$ by three dead layer thickness dependent parameters.

$$
E_{k i n}=p_{0}+p_{1} E_{d e p}+p_{2} E_{d e p}^{2}
$$




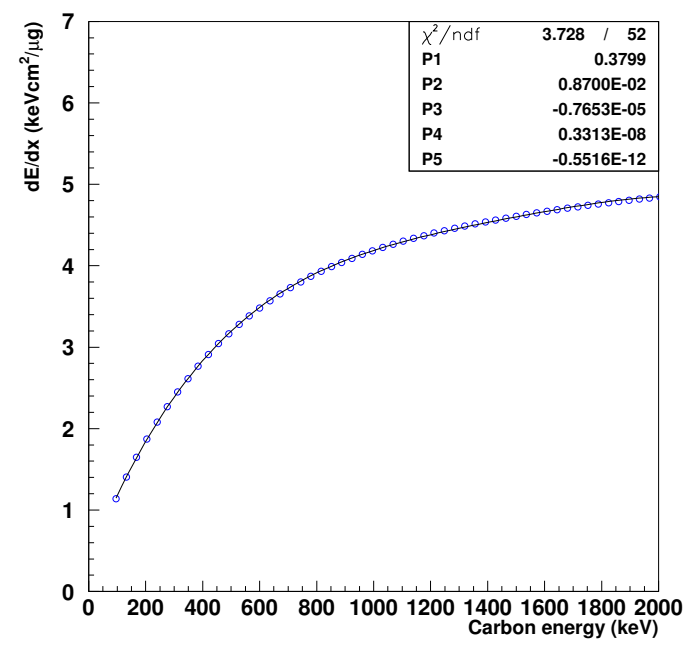

Figure A.1: Energy loss vs. incident energy for carbon in silicon as generated by MSTAR. The points are fit with a fourth order polynomial. The fit parameters are displayed in the upper-right corner of the plot.

where the $p_{n}$ all depend on the dead layer thickness. Several values of $p_{n}$ are generated for $15 \mu \mathrm{g} / \mathrm{cm}^{2} \leq t_{\text {dead }} \leq 65 \mu \mathrm{g} / \mathrm{cm}^{2}$. The $t_{\text {dead }}$ dependence of each $p_{n}$ is then fit with a second order polynomial.

$$
p_{n}\left(t_{\text {dead }}\right)=C_{n, 0}+C_{n, 1} t_{\text {dead }}+C_{n, 2} t_{\text {dead }}^{2}
$$

The 9 parameters, $C_{n, m}$, resulting from these fits can then be used to express $E_{k i n}$ in terms of the measured energy, $E_{d e p}$, and the dead layer thickness, $t_{\text {dead }}$. Now, the correlation between $E_{k i n}$ and tof is fit from the measured data with $t_{\text {dead }}$ being a free parameter.

The measured time of flight for each event also requires a correction that must be fit from the data. The timing is measured relative to an electronic clock signal, which is synchronized to the revolution frequency of the proton bunch in the AGS. There is a time offset between this clock and the actual time that the 


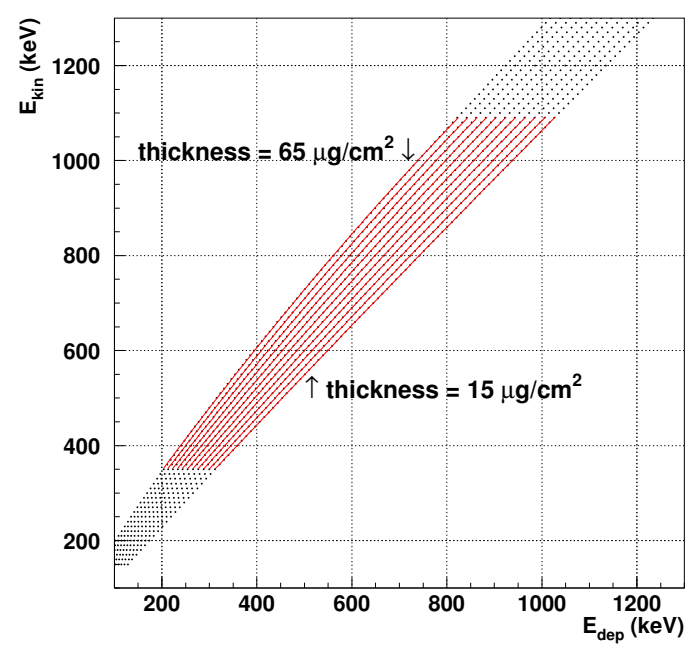

Figure A.2: Incident carbon energy versus energy deposited in the active silicon region from MSTAR calculations. Data are plotted for different dead layer thickness assumptions ranging from 15 to $65 \mu \mathrm{g} / \mathrm{cm}^{2}$. For each thickness the correlation is fit with a second order polynomial for $350 \mathrm{keV}<E_{\text {dep }}<1100 \mathrm{keV}$. 
proton bunch interacts with the carbon target, so the time of flight is given by

$$
\text { tof }=t_{\text {meas }}-t_{0}
$$

where $t_{\text {meas }}$ is the measured time and $t_{0}$ is the constant offset. Like $t_{\text {dead }}, t_{0}$ must be extracted from a fit to the correlation between $E_{k i n}$ and $t o f$. Also, $t_{0}$ can vary by a few ns for each channel of the SSDs due to differences in cable length.

Equations A.1 and A.3 relate $E_{k i n}$ and tof to two directly measured quantities: $E_{d e p}$ and $t_{m e a s}$. The deposited energy, $E_{d e p}$, is found by multiplying the measured signal amplitude $(A m p)$ by the alpha calibration constant, $C_{\alpha}$.

$$
C_{\alpha}=C_{a t t e n} \times \frac{E_{\alpha}}{\mu_{A D C}},
$$

where $E_{\alpha}$ is the alpha energy, $5486 \mathrm{keV}$, and $\mu_{A D C}$ is the mean from a gaussian fit to the alpha source ADC distribution. $C_{\text {atten }}$ is a factor to correct for the attenuation of the alpha signals. $14 \mathrm{~dB}$ attenuators were used to collect the alpha data, no attenuation was used for collecting carbon data for polarization measurements.

Substituting Equations A.1 and A.3 into the tof-energy correlation (Equation 1.1) yields the following relation:

$$
t_{\text {meas }}=l \sqrt{\frac{m_{C}}{2}} \frac{1}{\sqrt{p_{0}+p_{1} C_{\alpha} A m p+p_{2}\left(C_{\alpha} A m p\right)^{2}}}+t_{0} .
$$

Equations A.5 and A.2 are used to fit the correlation between the measured quantities $t_{m e a s}$ and $A m p$ while $t_{\text {dead }}$ and $t_{0}$ are free parameters. The parameters $t_{\text {dead }}$ and $t_{0}$ are extracted from a portion of the polarimeter measurements. These parameters are then used to reconstruct tof and $E_{k i n}$ for all the polarimeter data. 


\section{Appendix B: Asymmetry and Analyzing Power Formulae}

The asymmetries and effective analyzing power used for the AGS CNI polarimeter are defined here. The so-called square-root formula, as first described in Reference [6], is used to calculate asymmetries.

\section{B.1 Square-root Asymmetries}

The asymmetries are calculated from the number of measured events, which are measured directly using left and right detector arms. The number of measured events can be related to properties of the beam, the detectors, and the analyzing power for the process as follows:

$$
\begin{aligned}
N_{L}^{\uparrow} & =B^{\uparrow} d \Omega_{L}\left(1+P^{\uparrow} A_{N L}\right) \\
N_{L}^{\downarrow} & =B^{\downarrow} d \Omega_{L}\left(1-P^{\downarrow} A_{N L}\right) \\
N_{R}^{\uparrow} & =B^{\uparrow} d \Omega_{R}\left(1-P^{\uparrow} A_{N R}\right) \\
N_{R}^{\downarrow} & =B^{\downarrow} d \Omega_{R}\left(1+P^{\downarrow} A_{N R}\right),
\end{aligned}
$$

where $N_{L(R)}^{\uparrow(\downarrow)}$ is the number of events detected in the left (right) detector arm with the beam in the up (down) polarization state. The integrated beam intensity is $B^{\uparrow(\downarrow)}$, and the beam polarization is $P^{\uparrow(\downarrow)}$ for the up (down) polarization state. $d \Omega_{L(R)}$ is the solid angle times efficiency, and $A_{N L(R)}$ is the analyzing power for the left (right) detector arm. The mean values and asymmetries of these quantities 
are given by the expressions:

$$
\begin{aligned}
B & =\left(B^{\uparrow}+B^{\downarrow}\right) / 2 \\
\varepsilon_{B} & =\frac{B^{\uparrow}-B^{\downarrow}}{B^{\uparrow}+B^{\downarrow}} \\
P & =\left(P^{\uparrow}+P^{\downarrow}\right) / 2 \\
\varepsilon_{P} & =\frac{P^{\uparrow}-P^{\downarrow}}{P^{\uparrow}+P^{\downarrow}} \\
d \Omega & =\left(d \Omega_{L}+d \Omega_{R}\right) / 2 \\
\varepsilon_{\Omega} & =\frac{d \Omega_{L}-d \Omega_{R}}{d \Omega_{L}+d \Omega_{R}} \\
A_{N} & =\left(A_{N L}+A_{N R}\right) / 2 \\
\varepsilon_{A} & =\frac{A_{N L}-A_{N R}}{A_{N L}+A_{N R}} .
\end{aligned}
$$

The measured asymmetry used for the AGS CNI polarimeter is defined as

$$
\varepsilon=\frac{\sqrt{N_{R}^{\uparrow} N_{L}^{\downarrow}}-\sqrt{N_{R}^{\downarrow} N_{L}^{\uparrow}}}{\sqrt{N_{R}^{\uparrow} N_{L}^{\downarrow}}+\sqrt{N_{R}^{\downarrow} N_{L}^{\uparrow}}} .
$$

Two other asymmetries, $\varepsilon_{a c c}$ and $\varepsilon_{l u m}$, are also calculated. These are used to study the systematic effects from differences in the left and right detector acceptances and from differences in up and down polarization states. $\varepsilon_{a c c}$ and $\varepsilon_{l u m}$ are defined as

$$
\begin{aligned}
& \varepsilon_{\text {acc }}=\frac{\sqrt{N_{R}^{\uparrow} N_{R}^{\downarrow}}-\sqrt{N_{L}^{\uparrow} N_{L}^{\downarrow}}}{\sqrt{N_{R}^{\uparrow} N_{R}^{\downarrow}}+\sqrt{N_{L}^{\uparrow} N_{L}^{\downarrow}}}, \\
& \varepsilon_{l u m}=\frac{\sqrt{N_{R}^{\uparrow} N_{L}^{\uparrow}}-\sqrt{N_{R}^{\downarrow} N_{L}^{\downarrow}}}{\sqrt{N_{R}^{\uparrow} N_{L}^{\uparrow}}+\sqrt{N_{R}^{\downarrow} N_{L}^{\downarrow}}} .
\end{aligned}
$$

The statistical error of the asymmetries in Equations B.3, B.4, and B.5 is given by

$$
\sigma_{\varepsilon}=\frac{\sqrt{N_{R}^{\uparrow} N_{L}^{\downarrow}\left(N_{R}^{\downarrow}+N_{L}^{\uparrow}\right)+N_{R}^{\downarrow} N_{L}^{\uparrow}\left(N_{R}^{\uparrow}+N_{L}^{\downarrow}\right)}}{\left(\sqrt{N_{R}^{\uparrow} N_{L}^{\downarrow}}+\sqrt{N_{R}^{\downarrow} N_{L}^{\uparrow}}\right)^{2}}
$$


Note that if the asymmetries are small then the four event yields are all ap-

proximately equal, $N_{R}^{\uparrow} \approx N_{R}^{\downarrow} \approx N_{L}^{\uparrow} \approx N_{L}^{\downarrow}$. In this case, Equation B.6 reduces to

$$
\sigma_{\varepsilon} \approx \frac{1}{\sqrt{N_{t o t}}}
$$

where $N_{\text {tot }}=N_{R}^{\uparrow}+N_{R}^{\downarrow}+N_{L}^{\uparrow}+N_{L}^{\downarrow}$.

The measured asymmetries (Equations B.3, B.4, and B.5) are related to the quantities in Equations B.2 as follows:

$$
\begin{aligned}
\varepsilon & =P A_{N}\left[1-2\left(P A_{N}\right) \varepsilon_{P} \varepsilon_{A}\right]+\text { h.o.t. } \\
& \approx P A_{N} \\
\varepsilon_{a c c} & =\varepsilon_{\Omega}+\left(P A_{N}\right) \varepsilon_{P}+\text { h.o.t. } \\
& \approx \varepsilon_{\Omega} \\
\varepsilon_{l u m} & =\varepsilon_{B}+\left(P A_{N}\right) \varepsilon_{A}+\text { h.o.t. } \\
& \approx \varepsilon_{B} .
\end{aligned}
$$

\section{B.2 Effective Analyzing Power}

The analyzing power used for the AGS CNI polarimeter is based on a measurement from the E950 experiment [1]. E950 measured the analyzing power for $p C$ elastic scattering for a polarized proton beam of energy $21.7 \mathrm{GeV}$. The data from the E950 measurement are fit with theoretical constraints as described in Reference [2]. The values from this fit are extrapolated to predict the analyzing power for beam energies of $24.3 \mathrm{GeV}$ and $100 \mathrm{GeV}$. The model predicts a very small beam energy dependence for energies greater than $21.7 \mathrm{GeV}$.

The theoretical value of the analyzing power at $24.3 \mathrm{GeV}$ is used to determine 
Table B.1: Analyzing Power used for the AGS CNI Polarimeter during the 2003 Run for $E_{\text {beam }}=24.3 \mathrm{GeV}$.

\begin{tabular}{|c||c|c|c|c|c|}
\hline$A_{N}^{\text {th }}$ & 0.03445 & 0.02634 & 0.02039 & 0.01605 & 0.01271 \\
\hline$|t|\left[(\mathrm{GeV} / c)^{2}\right]$ & 0.0030 & 0.0055 & 0.0080 & 0.0105 & 0.0130 \\
\hline \hline$A_{N}^{\text {th }}$ & 0.01001 & 0.00778 & 0.00588 & 0.00423 & 0.00280 \\
\hline$|t|\left[(\mathrm{GeV} / c)^{2}\right]$ & 0.0155 & 0.0180 & 0.0205 & 0.0230 & 0.0255 \\
\hline
\end{tabular}

an effective analyzing power, $\left\langle A_{N}\right\rangle$, for the AGS CNI polarimeter. The effective analyzing power is calculated by weighting the theoretical values by the measured event yields.

$$
\left\langle A_{N}\right\rangle=\frac{\sum_{i=1}^{n_{b i n}}\left(A_{N}^{t h}\right)_{i} N_{i}}{\sum_{i=1}^{n_{b i n}} N_{i}},
$$

where $\left(A_{N}^{t h}\right)_{i}$ is the theoretical value of the analyzing power for the $i^{t h}-t$ bin. $N_{i}$ is the measured yield for the $i^{t h}$ bin, and $n_{b i n}$ is the number of $-t$ bins used for the measurement. The beam polarization, $P$, measured by the AGS CNI polarimeter is given by

$$
P=\varepsilon /\left\langle A_{N}\right\rangle
$$

The theoretical values of the analyzing power and $-t$ values used for the 2003 run are displayed in Table B.1. For the 2004 and 2005 runs, the analyzing power calculation was revised, resulting in a $\sim 10 \%$ change in the effective analyzing power. The revised analyzing power for beam energy $24.3 \mathrm{GeV}$ are shown in Table B.2. 
Table B.2: Analyzing Power used for the AGS CNI Polarimeter during the 2004 and 2005 Runs for $E_{\text {beam }}=24.3 \mathrm{GeV}$.

\begin{tabular}{|c||c|c|c|c|c|}
\hline$A_{N}^{\text {th }}$ & 0.03252 & 0.04021 & 0.03778 & 0.03399 & 0.03044 \\
\hline$|t|\left[(\mathrm{GeV} / c)^{2}\right]$ & 0.001 & 0.002 & 0.003 & 0.004 & 0.005 \\
\hline \hline$A_{N}^{\text {th }}$ & 0.02734 & 0.02466 & 0.02234 & 0.02030 & 0.01849 \\
\hline$|t|\left[(\mathrm{GeV} / c)^{2}\right]$ & 0.006 & 0.007 & 0.008 & 0.009 & 0.010 \\
\hline \hline$A_{N}^{\text {th }}$ & 0.01687 & 0.01541 & 0.01407 & 0.01285 & 0.01173 \\
\hline$|t|\left[(\mathrm{GeV} / c)^{2}\right]$ & 0.011 & 0.012 & 0.013 & 0.014 & 0.015 \\
\hline \hline$A_{N}^{\text {th }}$ & 0.01069 & 0.00973 & 0.00882 & 0.00798 & 0.00719 \\
\hline$|t|\left[(\mathrm{GeV} / c)^{2}\right]$ & 0.016 & 0.017 & 0.018 & 0.019 & 0.020 \\
\hline \hline$A_{N}^{\text {th }}$ & 0.00644 & 0.00574 & 0.00507 & 0.00444 & 0.00384 \\
\hline$|t|\left[(\mathrm{GeV} / c)^{2}\right]$ & 0.021 & 0.022 & 0.023 & 0.024 & 0.025 \\
\hline
\end{tabular}




\section{REFERENCES}

[1] J. Tojo, et al. Phys. Rev. Lett. 89, 052302 (2002) [arXiv:hep-ex/0206057].

[2] T.L. Trueman. hep-ph/0305085 (2003).

[3] W.R. Lozowski and J.D. Hudson. Nucl. Instrum. Meth. A 303, 34 (1991).

[4] D. Svirida, et al. Spin 2004, 16th International Spin Physics Symposium Conf. Proc. (2004).

[5] H. Paul and A. Schinner. Nucl. Instrum. Meth. B 195, 166-174 (2002). [Online] Available: http://www.exphys.unilinz.ac.at/Stopping/MstarWWW/MSTARInstr.htm.

[6] H. Spinka. Argonne Nat. Lab. Report ANL-HEP-PR-80-02 (1980). 